\title{
Congestion Games with Failures
}

\author{
Michal Penn* \\ mpenn@ie.technion.ac.il
}

\author{
Maria Polukarov*
pmasha@tx.technion.ac.il \\ Maria Polukarov*
pmasha@tx.technion.ac.il
}

10th July 2005

\author{
Moshe Tennenholtz* \\ moshet@ie.technion.ac.il
}

\begin{abstract}
We introduce a new class of games, congestion games with failures (CGFs), which extends the class of congestion games to allow for facility failures. In a CGF agents share a common set of facilities (service providers), where each service provider (SP) may fail with some known probability. For reliability reasons, an agent may choose a subset of the SPs in order to try and perform his task. The cost of an agent for utilizing any SP is an agent-specific function of the total number of agents using this SP. A main feature of this setting is that the cost for an agent for successful completion of his task is the minimum of the costs of his successful attempts. We show that although congestion games with failures do not admit a potential function, and thus are not isomorphic to classic congestion games, they always possess a pure-strategy Nash equilibrium. Moreover, an efficient algorithm for the construction of pure-strategy Nash equilibrium profile is presented. We also show that the SPs' congestion experienced in different Nash equilibria is (almost) unique. For the subclass of symmetric CGFs we give a characterization of best and worst Nash equilibria, present algorithms for their construction, and compare the social disutilities of the agents at these points.
\end{abstract}

\section{Introduction}

Rosenthal [10] introduced the class of congestion games and proved that they always possess a Nash equilibrium in pure strategies. Congestion games are noncooperative games in which a collection of agents have to choose from a finite set of alternatives (facilities). The utility of an agent from using a particular facility depends only on the number of agents using it, and his total utility is the sum of the utilities obtained from the facilities he uses. Congestion games have been used to model traffic behavior in road and communication networks, competition among firms for production processes, migration of animals between different habitats, and received a lot of attention in the recent computer science and electronic commerce communities [5, 8, 9, 10, 11]. Rosenthal [10] studied games with a finite number of players. More recently, several authors have considered nonatomic congestion games with a continuum of players $[5,6,13]$.

However, the above settings do not take into consideration the possibility that facilities may fail to execute their assigned tasks. Typically, the facilities are machines, computers, service providers, communication lines etc. These kinds of facilities are obviously prone to failures because of breakage or for any other reasons. Thus, the issue of failures should not be ignored.

${ }^{*}$ Faculty of Industrial Engineering and Management, Technion, Haifa, Israel 
As it turns out, such failures have significant implications on agent behavior, as illustrated by the following simple example. Consider a reliable network with two nodes $s$ and $t$, and two parallel links. Assume an agent wishes to send a message from $s$ to $t$. Then, he would send the message along one of the links. However, if the network links are not reliable then, for reliability reasons, the agent may decide to send his message along both links.

Suppose now that $n$ agents share a reliable network with two parallel links, where the cost associated with each link is a (nondecreasing) function $l(x)$ of the congestion experienced by this link. Each agent has to send a message from $s$ to $t$, and his aim is to minimize his own cost. If $n$ is even, then in an equilibrium, half of the agents would take one link and the other half would use the second link, and thus the cost to each agent is $l\left(\frac{n}{2}\right)$. If the network links are not reliable, the agents might send a message along both links. As a result of such behavior, the network might be overloaded, and the cost to each agent will be very high. Therefore, each agent wants to maximize the probability of successful delivery of his message and, simultaneously, to minimize his cost.

The above example illustrates the need for a careful study of the effects of failures in congestion settings. In order to address this challenge, we introduce a model for congestion games with failures (CGFs), and establish several basic results for this model. To the best of our knowledge, no attempt has been made so far to incorporate the issue of failures in congestion settings.

In a CGF agents share a common set of facilities (service providers), where each service provider (SP) may fail with some known probability. For reliability reasons, an agent may choose a subset of the service providers in order to try and perform his task. Therefore, each agent's set of pure strategies coincide with the power set of the set of SPs, and the total load on the system is not known in advance, but strategy-dependent. The cost for an agent for successful completion of his task is the minimum of the costs of his successful attempts. The cost function associated with each SP is not universal but agentspecific. That is, the utility to an agent depends not only on the number of agents using the same SP, but also on the identity of the agent in question. Congestion games with agent-specific cost functions were first studied by Milchtaich [4]. This generalization was, however, accompanied by the assumption that each agent chooses only one facility.

The main result of our work is that, although congestion games with failures do not admit a potential function, and thus are not isomorphic to classic congestion games, they always possess a pure-strategy Nash equilibrium. Moreover, our proof is constructive, and an efficient algorithm for the construction of pure-strategy Nash equilibrium is presented. We also show that the SPs' congestion experienced in different Nash equilibria is (almost) unique. For the subclass of symmetric CGFs we give a characterization of best and worst Nash equilibria, present algorithms for their construction, and compare the social disutilities of the agents at these points.

Furthermore, we consider the worst possible ratio between the social disutilities incurred by agents in an equilibrium and in an optimal outcome. This ratio (dubbed "the price of anarchy") was proposed by Koutsoupias and Papadimitriou [3] as a measure of the inefficiency of selfish behavior in noncooperative systems, and was extensively studied for nonatomic congestion games $[2,11,12,13]$. We show that in congestion games with failures the price of anarchy depends on the parameters of the game and cannot be bounded by a constant value as in classic congestion games, even for very simple (e.g., linear) cost functions.

The paper is organized as follows. In Section 2 we define our model. In Section 3 we 
show that CGFs do not admit a potential. In Section 4 we provide a (constructive) proof of the existence of pure-strategy Nash equilibrium in CGFs and consider its uniqueness properties. Section 5 is devoted to symmetric CGFs. We characterize the best and worst Nash equilibria in symmetric CGFs, present algorithms for their construction and provide an upper bound on the ratio between them. We also discuss the ratio between Nash equilibria and social optimum in these games.

\section{The Model}

A CGF is defined as follows. Let $N=\{1, \ldots, n\}$ be a finite set of agents, and let $E=$ $\{1, \ldots, m\}$ be a finite nonempty set of independent service providers, each associated with a failure probability. Each agent has a task which can be carried out by any of the service providers. Agent $i$ 's disutility from an uncompleted task is evaluated by his failure cost (denoted by $W_{i}$ ). The service cost (denoted by $l_{e}^{i}$ ) for agent $i$ for utilizing service provider $e$ consists of an execution cost (denoted by $b_{e}^{i}$ ) and a fixed completion cost (denoted by $\left.a\right) .{ }^{1}$ The disutility $\pi_{i}$ of agent $i$ from a combination of strategies (one for each agent) is the expectation of the sum of his failure and service costs, where the service cost for an agent is the minimum of the service costs of the SPs he has chosen which did not fail. This is defined more precisely below.

The success probability of $e \in E$ is denoted by $s_{e}\left(0<s_{e}<1\right)$. Similarly, $f_{e}=1-s_{e}$ stands for the failure probability of $e$. The set of pure strategies $\Sigma_{i}$ for agent $i \in N$ is the power set of the set of SPs: $\Sigma_{i}=\mathcal{P}(E)$, and the set of pure-strategy profiles is defined to be $\Sigma=\Sigma_{1} \times \cdots \times \Sigma_{n}=[\mathcal{P}(E)]^{n}$.

Let $\sigma=\left(\sigma_{1}, \ldots, \sigma_{n}\right) \in \Sigma$ be a combination of pure strategies. The (|E|-dimensional) congestion vector that corresponds to $\sigma$ is $h^{\sigma}=\left(h_{e}^{\sigma}\right)_{e \in E}$, where $h_{e}^{\sigma}=\left|\left\{i \in N \mid e \in \sigma_{i}\right\}\right|$. The execution cost of service provider $e$ for agent $i$ is a function $b_{e}^{i}: \Sigma \rightarrow \mathbb{R}$ of the congestion experienced by $e$. The disutility function of agent $i, \pi_{i}: \Sigma \rightarrow \mathbb{R}$, is defined as follows. If agent $i$ chooses strategy $\sigma_{i}=\varnothing$ (i.e., does not assign his task to any service provider) then his disutility equals his failure cost, $\pi_{i}(\sigma)=W_{i}$. For any strategy $\sigma_{i} \neq \varnothing$ of agent $i$,

$$
\begin{aligned}
\pi_{i}(\sigma) & =W_{i} \prod_{e \in \sigma_{i}} f_{e}+\sum_{A \in \mathcal{P}\left(\sigma_{i}\right) \backslash\{\varnothing\}} \min _{e \in A}\left(b_{e}^{i}\left(h_{e}^{\sigma}\right)+a\right) \prod_{e \in A} s_{e} \prod_{e \in \sigma_{i} \backslash A} f_{e} \\
& =W_{i} \prod_{e \in \sigma_{i}} f_{e}+\sum_{A \in \mathcal{P}\left(\sigma_{i}\right) \backslash\{\varnothing\}} \min _{e \in A} l_{e}^{i}\left(h_{e}^{\sigma}\right) \prod_{e \in A} s_{e} \prod_{e \in \sigma_{i} \backslash A} f_{e},
\end{aligned}
$$

where $a$ is the fixed completion cost, $b_{e}^{i}\left(h_{e}^{\sigma}\right)$ is the execution cost of service provider $e$ for agent $i$, when its congestion is $h_{e}^{\sigma}$, and the sum of execution and fixed completion costs $l_{e}^{i}\left(h_{e}^{\sigma}\right)=b_{e}^{i}\left(h_{e}^{\sigma}\right)+a$ is the service cost of service provider $e$ for agent $i$.

We assume that $b_{e}^{i}(\cdot)$ is a nonnegative nondecreasing function satisfying $b_{e}^{i}(x) \leq W_{i}$ for all $i \in N, e \in E$ and integer $0 \leq x \leq n$. This means that the execution of a task does not cost more than its failure. W.l.o.g., we also assume that for any agent $i$ his failure cost $W_{i}$ is larger than the fixed completion cost $a$. Otherwise, the obvious dominant strategy of agent $i$ is to avoid assigning his task to any service provider. Note that for all $0 \leq x \leq n$, $b_{e}^{i}(x) \leq W_{i}$ and $a \leq W_{i}$, but $l_{e}^{i}(x)$ might be larger than $W_{i}$. Obviously, if $l_{e}^{i}(1)>W_{i}$ for all $e \in E$, the dominant strategy of agent $i$ is to avoid assigning a task, i.e. in this case

\footnotetext{
${ }^{1}$ This models for example a payment to the network administrator for successful execution of a task, by one or more of the service providers. Our model can be extended, while leading to similar results, to the case where the completion cost is agent-dependent or facility dependent.
} 
agent $i$ can be actually ignored. Therefore, w.l.o.g., we assume that such cases do not take place.

\section{CGFs have no potential}

Monderer and Shapley [7] introduced the notion of potential function and defined a potential game to be a game which possesses a potential function. A potential function is a real-valued function over the set of pure-strategy profiles, with the property that the gain (or loss) of an agent shifting to another strategy is equal to the corresponding increment of the potential function. The authors showed that the classes of potential games and congestion games coincide.

In this section we show that the class of CGFs does not possess a potential function, and therefore is not isomorphic to the class of congestion games. Notice that congestion games with agent-specific cost functions, introduced by Milchtaich [4], can be viewed as CGFs with zero failure probabilities. It has been shown that such games are not potential games. Nevertheless, our result for CGFs is more general. We show that even agent-symmetric CGFs do not admit a potential function. Hence, the non-existence of a potential in CGFs is a result of allowing facility failures. To prove this statement we employ Theorem 3.1 of Monderer and Shapley [7]. First, however, we need to present some definitions.

A path in $\Sigma$ is a sequence $\gamma=\left(\sigma^{0} \rightarrow \sigma^{1} \rightarrow \cdots\right)$ such that for every $k \geq 1$ there exists a unique player, say player $i$, such that $\sigma^{k}=\left(\sigma_{-i}^{k-1}, x\right)$ for some $x \neq \sigma_{i}^{k-1}$ in $\Sigma_{i}$. $\sigma^{0}$ is called the initial point of $\gamma$, and if $\gamma$ is finite, then its last element is called the terminal point of $\gamma$. A finite path $\gamma=\left(\sigma^{0} \rightarrow \sigma^{1} \rightarrow \cdots \rightarrow \sigma^{K}\right)$ is closed if $\sigma^{0}=\sigma^{K}$. It is a simple closed path if in addition $\sigma^{l} \neq \sigma^{k}$ for every $0 \leq l \neq k \leq K-1$. The length of a simple closed path is defined to be the number of distinct points in it; that is, the length of $\gamma=\left(\sigma^{0} \rightarrow \sigma^{1} \rightarrow \cdots \rightarrow \sigma^{K}\right)$ is $K$. For a finite path $\gamma=\left(\sigma^{0} \rightarrow \sigma^{1} \rightarrow \cdots \rightarrow \sigma^{K}\right)$ and for a vector $U=\left(U_{1}, \ldots, U_{n}\right)$ of utility functions, let us define

$$
U(\gamma)=\sum_{k=1}^{K}\left[U_{i_{k}}\left(\sigma^{k}\right)-U_{i_{k}}\left(\sigma^{k-1}\right)\right],
$$

where $i_{k}$ is the unique deviator at step $k$. Then,

Theorem 3.1 (Monderer-Shapley, [7]) Let $\Gamma$ be a game in strategic form. Then, $\Gamma$ is a potential game if and only if $U(\gamma)=0$ for every finite simple closed path $\gamma$ of length 4.

By this theorem, if $\Gamma$ is a game in strategic form with $U_{i}: \Sigma \rightarrow \mathbb{R}$ the utility function of agent $i$, then $\Gamma$ is a potential game if and only if for every $i, j \in N$, for every $z \in \Sigma_{-\{i, j\}}$, and for every $x_{i}, y_{i} \in \Sigma_{i}$ and $x_{j}, y_{j} \in \Sigma_{j}$,

$$
U_{i}(\beta)-U_{i}(\alpha)+U_{j}(\gamma)-U_{j}(\beta)+U_{i}(\delta)-U_{i}(\gamma)+U_{j}(\alpha)-U_{j}(\delta)=0,
$$

where $\alpha=\left(x_{i}, x_{j}, z\right), \beta=\left(y_{i}, x_{j}, z\right), \gamma=\left(y_{i}, y_{j}, z\right), \delta=\left(x_{i}, y_{j}, z\right)$ (thus, $\alpha \rightarrow \beta \rightarrow \gamma \rightarrow$ $\delta \rightarrow \alpha$ is a simple closed path of length 4$)$.

Proposition 3.2 The class of CGFs does not possess a potential function.

Proof: A counterexample is the following symmetric game $G$ in which two agents $(N=\{1,2\})$ wish to assign a task to two independent SPs $\left(E=\left\{e_{1}, e_{2}\right\}\right)$. The failure probability $f$ 
of each SP is positive $(f>0)$. The failure cost to each of the agents is $W \geq 2$, and the service cost function of each SP to each agent is given by $l_{e}^{i}(x)=\min \{x, W\}+a(\forall e, i)$. Consider the simple closed path of length 4 which is formed by

$$
\alpha=\left(\varnothing,\left\{e_{2}\right\}\right), \beta=\left(\left\{e_{1}\right\},\left\{e_{2}\right\}\right), \gamma=\left(\left\{e_{1}\right\},\left\{e_{1}, e_{2}\right\}\right), \delta=\left(\varnothing,\left\{e_{1}, e_{2}\right\}\right) \text { : }
$$

\begin{tabular}{|c|c|c|}
\hline & $\left\{e_{2}\right\}$ & $\left\{e_{1}, e_{2}\right\}$ \\
\hline$\varnothing$ & $\pi_{1}=W$ & $\pi_{1}=W$ \\
& $\pi_{2}=f W+(1-f)(\min \{1, W\}+a)$ & $\pi_{2}=f^{2} W+\left(1-f^{2}\right)(\min \{1, W\}+a)$ \\
\hline$\left\{e_{1}\right\}$ & $\pi_{1}=f W+(1-f)(\min \{1, W\}+a)$ & $\pi_{1}=f W+(1-f)(\min \{2, W\}+a)$ \\
& $\pi_{2}=f W+(1-f)(\min \{1, W\}+a)$ & $\pi_{2}=f^{2} W+(1-f)(\min \{1, W\}+a)+f(1-f)(\min \{2, W\}+a)$ \\
\hline
\end{tabular}

Then,

$$
\begin{aligned}
& \pi_{1}(\beta)-\pi_{1}(\alpha)+\pi_{2}(\gamma)-\pi_{2}(\beta)+\pi_{1}(\delta)-\pi_{1}(\gamma)+\pi_{2}(\alpha)-\pi_{2}(\delta) \\
& =(f W+(1-f)(\min \{1, W\}+a))-W \\
& +\left(f^{2} W+(1-f)(\min \{1, W\}+a)+f(1-f)(\min \{2, W\}+a)\right) \\
& -(f W+(1-f)(\min \{1, W\}+a))+W-(f W+(1-f)(\min \{2, W\}+a)) \\
& +(f W+(1-f)(\min \{1, W\}+a))-\left(f^{2} W+\left(1-f^{2}\right)(\min \{1, W\}+a)\right) \\
& =-(1-f)^{2}(\min \{2, W\}-\min \{1, W\})=-(1-f)^{2} \neq 0 .
\end{aligned}
$$

Then, by Theorem 3.1, congestion games with failures do not possess a potential function.

\section{Pure-strategy Nash equilibria in CGFs}

By Monderer and Shapley [7], every finite potential game possesses a pure-strategy Nash equilibrium. We have shown in Section 3 that CGFs do not admit a potential function, but this fact, in general, does not contradict the existence of an equilibrium in pure strategies. In this section we present the main contribution of this paper. We prove that all congestion games with failures possess Nash equilibria in pure strategies, and present an efficient algorithm that finds such equilibrium points in a given CGF. Moreover, we show that different Nash equilibrium profiles of a given CGF correspond to (almost) the same congestion vector.

\subsection{Existence and construction}

The following theorem is the main result of this paper.

Theorem 4.1 Congestion games with failures possess a Nash equilibrium in pure strategies.

The proof of Theorem 4.1 is quite long and tedious, and is given in the Appendix. One point to notice is that the proof is constructive and makes use of the following efficient simple algorithm for finding a pure Nash equilibrium in a given CGF. 


\subsubsection{NE-algorithm}

Initialization: For all $1 \leq i \leq n$, set $\sigma_{i}:=\varnothing$.

Main step: $\quad$ For all $e \in E$ :

(1) Sort the agents in a non-increasing order of $x_{e}^{i}=\max \left\{x \mid W_{i}>l_{e}^{i}(x), x=0,1, \ldots, n\right\}$.

Let $\varphi_{e}: N \rightarrow\{1, \ldots, n\}$

$i \mapsto i_{e}=\varphi_{e}(i)$

be the corresponding permutation function.

(2) For $i_{e}=1$ to $n$ :

if $i_{e} \leq x_{e}^{i}$, then $\sigma_{i}:=\sigma_{i} \cup\{e\}$.

\section{2 (Almost) uniqueness}

We consider uniqueness properties of Nash equilibria in CGFs. We restrict our attention to games with strictly increasing service cost functions, and show that in such CGFs the difference between the congestion experienced by an SP in two different Nash equilibria is bounded by 1 .

Let $N E \subseteq \Sigma$ be a set of Nash equilibrium pure-strategy combinations, and let $h_{M}$ represent the maximal congestion that may be experienced by any service provider at any Nash equilibrium, i.e. $h_{M}=\max \left\{h_{e}^{\sigma} \mid e \in E, \sigma \in N E\right\}$. Then,

Proposition 4.2 If for all $e \in E$ and $i \in N, l_{e}^{i}(x)$ is a strictly increasing monotone function on the interval $0 \leq x \leq h_{M}$, then for any pair of Nash equilibrium strategy profiles $\sigma^{1}, \sigma^{2} \in N E$ the inequality $\left|h_{e}^{\sigma^{1}}-h_{e}^{\sigma^{2}}\right| \leq 1$ holds for all $e \in E$.

For the proof of Proposition 4.2 we need the following claim.

Claim 4.3 Let $\sigma \in N E$ be a Nash equilibrium strategy profile. Then, for all $i \in N$,

$$
\begin{array}{lll}
\text { (i) } \quad l_{e}^{i}\left(h_{e}^{\sigma}\right) \leq W_{i}, & \forall e \in \sigma_{i} ; \\
\text { (ii) } \quad l_{e}^{i}\left(h_{e}^{\sigma}+1\right) \geq W_{i}, & \forall e \notin \sigma_{i} .
\end{array}
$$

The proof of Claim 4.3 is given in the Appendix.

Proof of Proposition 4.2: Let $\sigma^{1}, \sigma^{2} \in N E$ be Nash equilibrium strategy profiles, and assume that $h_{e}^{\sigma^{1}}>h_{e}^{\sigma^{2}}+1$ for some $e \in E$. Then, there is an agent $i$ such that $e \in \sigma_{i}^{1}$, but $e \notin \sigma_{i}^{2}$. By Claim 4.3, for agent $i$ we have $l_{e}^{i}\left(h_{e}^{\sigma^{1}}\right) \leq W_{i}$ and $l_{e}^{i}\left(h_{e}^{\sigma^{2}}+1\right) \geq W_{i}$. Therefore, $l_{e}^{i}\left(h_{e}^{\sigma^{1}}\right) \leq l_{e}^{i}\left(h_{e}^{\sigma^{2}}+1\right)$. Now, $h_{e}^{\sigma^{1}}>h_{e}^{\sigma^{2}}+1$ coupled with the monotonicity of $l_{e}^{i}(x)$ lead to $l_{e}^{i}\left(h_{e}^{\sigma^{1}}\right)>l_{e}^{i}\left(h_{e}^{\sigma^{2}}+1\right)$, in contradiction to Claim 4.3.

It is easy to show that if in addition to the requirements of Proposition 4.2, the cost function $l_{e}^{i}(\cdot)$ satisfies $l_{e}^{i}(x) \neq W_{i}$ for $0 \leq x \leq h_{M}$, then all Nash equilibria of a given CGF correspond to the same congestion vector, i.e. the congestion of any SP is fixed for all equilibrium points. In particular, all generic CGFs have this uniqueness property.

\section{Symmetric CGFs}

In this subsection we give some additional characterization of Nash equilibria in symmetric CGFs. In symmetric CGFs, the agents and the SPs are symmetric, i.e. for all $i=1, \ldots, n$ and $e \in E$ we have $W_{i}=W, f_{e}=f$, and $l_{e}^{i}(x)=l(x)$, for all $x \in\{0,1, \ldots, n\}$. We also present efficient algorithms for finding best and worst Nash equilibria, and make a comparison between this equilibria. 
Proposition 5.1 Let $G$ be a symmetric $C G F$. If $l(x)$ is a strictly increasing monotone function on the interval $0 \leq x \leq h_{M}$, then at any Nash equilibrium $\sigma \subseteq N E(G)$, the difference between the congestions of different SPs is bounded by 1, i.e. for all $\sigma \in N E$ and for all $a, b \in E$, the inquality $\left|h_{a}^{\sigma}-h_{b}^{\sigma}\right| \leq 1$ holds.

The proof of Proposition 5.1 is given in the Appendix.

\subsection{Best and worst equilibria}

Given a strategy profile $\sigma$, define the social disutility $\pi(\sigma)$ as the sum of the agents' disutilities in this strategy profile: $\pi(\sigma)=\sum_{i \in N} \pi_{i}(\sigma)$. A strategy profile that minimizes the social disutility over the set of strategy profiles is called a social optimum. A best (worst) equilibrium is a strategy profile that minimizes (maximizes) the social disutility over the set of equilibrium strategies. The social disutility in a best equilibrium describes the best result that can be obtained in a system with noncooperative selfish agents. The ratio between the social disutilities in a worst equilibrium and in a social optimum serves as a measure of the inefficiency of Nash equilibrium. In this subsection we characterize, construct and compare best and worst Nash equilibria in symmetric CGFs.

Proposition 5.2 Let $h^{*}=\max \{x \mid l(x)<W\}$. Then, there is a best Nash equilibrium strategy profile $\sigma$ in which the congestion on each $e \in E$ is $h_{e}^{\sigma}=h^{*}$, and moreover, ||$\sigma_{i}|-| \sigma_{j}|| \leq 1$ for all $i, j \in N$.

We prove below that the following algorithm (5.1.1), which is a (modified) version of the NE-algorithm (4.1.1), finds a best pure-strategy Nash equilibrium with the properties described by Proposition 5.2, in a given symmetric CGF.

\subsubsection{BNE-algorithm}

Initialization: For all $1 \leq i \leq n$, set $\sigma_{i}:=\varnothing$.

Main step: $\quad$ For all $e \in E$ :

(1) Sort the agents in an order

$$
\begin{aligned}
\varphi_{e}: N & \rightarrow\{1, \ldots, n\} \\
i & \mapsto i_{e}=\varphi_{e}(i)
\end{aligned}
$$

satisfying the following condition:

for all $i, j \in N,\left|\sigma_{i}\right|<\left|\sigma_{j}\right| \Rightarrow i_{e}=\varphi_{e}(i)<\varphi_{e}(j)=j_{e}$.

(2) Let $x_{\max }=\max \{x \mid W>l(x), x=0,1, \ldots, n\}$.

For $i_{e}=1$ to $n$ :

if $i_{e} \leq x_{\max }$, then $\sigma_{i}:=\sigma_{i} \cup\{e\}$.

Proof of Proposition 5.2: By Theorem 4.1, the combination of strategies constructed by BNE-algorithm is a Nash equilibrium strategy profile. One can check that the resulting combination of strategies satisfies the conditions of Proposition 5.2. More precisely, $x$ agents choose $\left\lfloor\frac{m h^{*}}{n}\right\rfloor$ service providers, where $m$ denote the number of SPs, and $y$ agents choose $\left\lfloor\frac{m h^{*}}{n}\right\rfloor+1$ service providers, where $x$ and $y$ satisfy the following equation:

$$
\left\{\begin{array}{lll}
x\left\lfloor\frac{m h^{*}}{n}\right\rfloor+y\left(\left\lfloor\frac{m h^{*}}{n}\right\rfloor+1\right) & =m h^{*} \\
x+y & =n .
\end{array}\right.
$$


The values of $x$ and $y$ are

$$
\begin{aligned}
& x=n\left(\left\lfloor\frac{m h^{*}}{n}\right\rfloor+1\right)-m h^{*} ; \\
& y=m h^{*}-n\left\lfloor\frac{m h^{*}}{n}\right\rfloor .
\end{aligned}
$$

Note that if $n$ divides $m h^{*}$, then $x=n, y=0$. To complete the proof we need the following two claims.

Claim 5.3 Let $\sigma \in N E$ be a combination of strategies at Nash equilibrium with two agents $i, j \in N$, such that $\left|\sigma_{i}\right|>\left|\sigma_{j}\right|+1$. Then, the combination of strategies

$$
\hat{\sigma}=\left(\sigma_{1}, \ldots, \sigma_{i} \backslash\{b\}, \ldots, \sigma_{j} \cup\{b\}, \ldots, \sigma_{n}\right),
$$

where $b \in \arg \max _{e \in \sigma_{i} \backslash \sigma_{j}} l\left(h_{e}^{\sigma}\right)$, is better than $\sigma$, i.e. $\sum_{k=1}^{n} \pi_{k}(\hat{\sigma}) \leq \sum_{k=1}^{n} \pi_{k}(\sigma)$.

Claim 5.4 Let $\sigma \neq(E, \ldots, E)$ be a Nash equilibrium strategy profile and let $i$ be an agent playing $\sigma_{i} \neq E$. Then, for all $k \in N$ and for all $e \in E \backslash \sigma_{i}$,

$$
\pi_{k}(\sigma) \leq \pi_{k}\left(\sigma_{1}, \ldots, \sigma_{i} \cup\{e\}, \ldots, \sigma_{n}\right) .
$$

The proofs of Claims 5.3 and 5.4 are given in the Appendix. By Claims 5.3 and 5.4, the combination of strategies constructed by the BNE-algorithm is a best Nash equilibrium profile.

The BNE-algorithm provides an efficient procedure for construction best Nash equilibria in symmetric CGFs, as defined in Proposition 5.2. Next we identify some worst equilibria in symmetric CGFs. These equilibrium points have very simple form and can be easily constructed, as follows from the next proposition.

Proposition 5.5 Let $h^{* *}=\arg \max \{x \mid l(x) \leq W\}$. Then, there is a worst Nash equilibrium strategy profile $\sigma$ in which exactly $h^{* *}$ agents play $E, n-h^{* *}$ agents play $\varnothing$ and $h_{e}^{\sigma}=h^{* *}$ for all $e \in E$.

The proof of Proposition 5.5 is given in the Appendix. Next we compare the best and worst Nash equilibria. Let us denote the social disutility of a best Nash equilibrium strategy profile by $\pi_{B}$, and the worst one by $\pi_{W}$ :

$$
\begin{aligned}
\pi_{W}= & h^{* *}\left(W f^{m}+l\left(h^{* *}\right)\left(1-f^{m}\right)\right)+\left(n-h^{* *}\right) W \\
= & h^{* *}\left(1-f^{m}\right)\left(l\left(h^{* *}\right)-W\right)+n W \\
\pi_{B}= & x\left(W f^{\left\lfloor\frac{m h^{*}}{n}\right\rfloor}+l\left(h^{*}\right)\left(1-f^{\left\lfloor\frac{m h^{*}}{n}\right\rfloor}\right)\right) \\
& +y\left(W f^{\left\lfloor\frac{m h^{*}}{n}\right\rfloor+1}+l\left(h^{*}\right)\left(1-f^{\left\lfloor\frac{m h^{*}}{n}\right\rfloor+1}\right)\right) \\
= & f^{\left\lfloor\frac{m h^{*}}{n}\right\rfloor}(x+f y)\left(W-l\left(h^{*}\right)\right)+n l\left(h^{*}\right),
\end{aligned}
$$

where $x$ and $y$ are given by (2).

Therefore, the ratio between social disutilities in worst and best equilibria is

$$
\frac{\pi_{W}}{\pi_{B}}=\frac{h^{* *}\left(1-f^{m}\right)\left(l\left(h^{* *}\right)-W\right)+n W}{f^{\left\lfloor\frac{m h^{*}}{n}\right\rfloor}(x+f y)\left(W-l\left(h^{*}\right)\right)+n l\left(h^{*}\right)} .
$$


Since $l\left(h^{*}\right)<W$ and $l\left(h^{* *}\right) \leq W$, we have that

$$
\frac{\pi_{W}}{\pi_{B}}<\frac{n W}{n l\left(h^{*}\right)}=\frac{W}{l\left(h^{*}\right)} .
$$

This implies that the values of the social disutility in different Nash equilibrium points lie in a very narrow range. In the context of social performance of Nash equilibria, one has to ask how far these values are from the social optimum.

\subsection{Nash equilibria and social optimum}

In this subsection we discuss the social performance of Nash equilibrium in CGFs. By Ashlagi [1], a best equilibrium strategy profile in classic congestion games with monotone concave cost functions is socially optimal. Simple examples (that were omitted from this paper) show that in CGFs with such cost functions, best equilibrium strategy profiles are not always socially optimal.

Furthermore, we show below that in CGFs the price of anarchy (the ratio between social disutilities in a worst Nash equilibrium and a social optimum) depends on the parameters of the game and cannot be bounded by a constant value as in classic congestion games, even for very simple (e.g., linear) cost functions.

Consider the following example. Suppose we have $n \geq 2$ agents sharing the set $E=$ $\{1, \ldots, m\}$ of $m \geq 2$ independent SPs. Each service provider $e \in E$ has the failure probability $f$, and the service cost of each SP for each agent is $l(x)=\min \{x, W\}+a$, where $a$ is a fixed completion cost. The failure cost of each agent is $W=n+a$.

The worst Nash equilibrium in this case corresponds to the combination of strategies $\sigma$ in which each agent chooses to use each of the SPs. The disutility of agent $i, i \in N$, at this point is

$$
\begin{aligned}
\pi_{i}(\sigma) & =f^{m} W+\left(1-f^{m}\right)(\min \{x, W\}+a) \\
& =f^{m}(n+a)+\left(1-f^{m}\right)(n+a)=n+a,
\end{aligned}
$$

and the social disutility is

$$
\pi(\sigma)=\sum_{i=1}^{n} \pi_{i}(\sigma)=n(n+a) .
$$

Consider the combination of strategies $\hat{\sigma}$ that corresponds to the following agents' behavior: each agent chooses only one SP and the agents divide up the SPs in a uniform way, i.e. each SP is chosen by $\frac{n}{m}$ agents (assume $m$ divides $n$ ). The disutility of agent $i, i \in N$, at this point is

$$
\begin{aligned}
\pi_{i}(\hat{\sigma}) & =f W+(1-f)\left(\min \left\{\frac{n}{m}, W\right\}+a\right) \\
& =f(n+a)+(1-f)\left(\frac{n}{m}+a\right)=f n+(1-f) \frac{n}{m}+a,
\end{aligned}
$$

and the social disutility is

$$
\pi(\hat{\sigma})=\sum_{i=1}^{n} \pi_{i}(\hat{\sigma})=n\left(f n+(1-f) \frac{n}{m}+a\right) .
$$


Then, the ratio between outcomes of the worst Nash equilibrium and the social optimum is

$$
\begin{aligned}
\frac{\pi(\sigma)}{\pi(O P T)} & \geq \frac{\pi(\sigma)}{\pi(\hat{\sigma})}=\frac{n(n+a)}{n\left(f n+(1-f) \frac{n}{m}+a\right)}=\frac{n+a}{f n+(1-f) \frac{n}{m}+a} \\
& =\frac{m(n+a)}{f m n+(1-f) n+a m} \stackrel{f \rightarrow 0}{\longrightarrow} \frac{m(n+a)}{n+a m} \stackrel{a \rightarrow 0}{\longrightarrow} \frac{m n}{n}=m .
\end{aligned}
$$

This implies that the price of anarchy in congestion games with failures, unlike in classic congestion games, is not bounded by a constant value, but is game-dependent.

\section{Discussion \& future work}

In this paper we studied congestion games in which facilities may fail to complete their assigned tasks. We have shown that these games do not admit a potential function, and therefore are not isomorphic to classic congestion games. However, we were able to prove the existence of pure-strategy Nash equilibrium for these games, and to find an efficient algorithm for its construction. We also showed that the congestion experienced by each of the facilities in different Nash equilibria is (almost) unique. For symmetric CGFs we provided a characterization of the best and worst Nash equilibria, presented algorithms for their construction, and made a comparison of agents' payoffs at these equilibrium points.

Since it is known that Nash equilibria do not optimize the overall welfare, the social performance of Nash equilibria should be studied. In this context, we outline the following two directions: (i) evaluation of the inefficiency of Nash equilibria; (ii) developing methods for improving the outcome of Nash equilibria. In both directions we have some partial results for the games presented in this paper. For instance, the price of anarchy in CGFs is a function of the parameters of the game and cannot be bounded by constant value as in classic congestion games, even for very simple (e.g., linear) cost functions. The inefficiency of Nash equilibria motivates the study of methods for improving the social outcome obtained by selfish agents. In this context, we have some positive results (that were omitted from this paper) showing that economic incentives, e.g. taxation, can improve the outcome of Nash equilibria in CGFs. That is, we can price the facilities to reduce the total social disutility of Nash equilibrium - the sum of the agents' disutilities plus taxes paid. We are interested in formulating meaningful conditions under which taxes can reduce the total cost of Nash equilibrium in games with failures.

As part of our research we plan to take further look at the modelling of noncooperative games with failures. The model we presented here could be extended or modified. In particular, the facility failures might be congestion-dependent or unknown to the agents.

Overall, we believe this work tackles a fundamental connection between distributed computing and game theory. While congestion is substantial to both disciplines (and indeed is extensively studied by both communities), the notion of selfish behavior pertains to game theory and the notion of failures originates from distributed computing. However, there is a natural connection between these topics which to the best of our knowledge is first explored in this work. 


\section{References}

[1] I. Ashlagi "The Value of Correlation in Strategic Form Games", M.Sc. Thesis, Technion - IIT, 2004.

[2] J.R. Correa, A.S. Schulz, N.E. Stier Moses "Computational Complexity, Fairness, and the Price of Anarchy of the Maximum Latency Problem", MIT Sloan School of Management, Working Paper 4447-03, November 2003.

[3] E. Koutsoupias, C. Papadimitriou "Worst-case Equilibria", In Proceedings of the 16th Annual Symposium on Theoretical Aspects of Computer Science, (1999), pp. 404-413.

[4] I. Milchtaich "Congestion Games with Player-Specific Payoff Functions", Games and Economic Behavior, Vol. 13, (1996), pp. 111-124.

[5] I. Milchtaich "Congestion Models of Competition", American Naturalist, Vol. 147, No. 5, (1996), pp. 760-783.

[6] I. Milchtaich "Social Optimality and Cooperation in Nonatomic Congestion Games", Journal of Economic Theory, Vol. 114, (2004), pp. 56-87.

[7] D. Monderer, L.S. Shapley "Potential Games", Games and Economic Behavior, Vol. 14, (1996), pp. 124-143.

[8] A. Orda, R. Rom, N. Shimkin "Competitive routing in multi-user communication networks", IEEE/ACM Transactions on Networking, Vol. 1, (1993), pp. 510-521.

[9] T. Quint, M. Shubik "A Model of Migration", Cowles Foundation Discussion Papers, No. 1088, Yale University, 1994.

[10] R.W. Rosenthal "A Class of Games Possessing Pure-Strategy Nash Equilibria", International Journal of Game Theory, Vol. 2, (1973), pp. 65-67.

[11] T. Roughgarden, E. Tardos, "How Bad is Selfish Routing", Journal of the ACM, Vol. 49, No. 2, (2002), pp. 236-259. Preliminary version in FOCS'00.

[12] T. Roughgarden, E. Tardos, "The Price of Anarchy is Independent of the Network Topology", In Proceedings of the 34th Annual ACM Symposium on the Theory of Computing, May 2002, pp. 428-437.

[13] T. Roughgarden, E. Tardos, "Bounding the Inefficiency of Equilibria in Nonatomic Congestion Games", Games and Economic Behavior, to appear.

\section{Appendix}

Notations: For any subset $A \subseteq E$ of the given set of SPs, we denote by $P(A)$ the set of all nonempty subsets of $A$, thus $P(A)=\mathcal{P}(A) \backslash\{\varnothing\}$. We denote by $l_{A}^{i}\left(h_{A}^{\sigma}\right)$ the minimal cost of the SPs in $A$ corresponding to congestion vector $h^{\sigma}: l_{A}^{i}\left(h_{A}^{\sigma}\right)=\min _{e \in A} l_{e}^{i}\left(h_{e}^{\sigma}\right)$. Similarly, we denote by $s(A)$ the success probability of all SPs in $A$, and by $f(A)$ the failure probability of all SPs in $A$. Then, the disutility function $\pi_{i}(\sigma)$ of agent $i$ choosing a nonempty strategy $\sigma_{i} \neq \varnothing$ may be written as follows:

$$
\pi_{i}(\sigma)=W_{i} f\left(\sigma_{i}\right)+\sum_{A \in P\left(\sigma_{i}\right)} l_{A}^{i}\left(h_{A}^{\sigma}\right) s(A) f\left(\sigma_{i} \backslash A\right),
$$


where

$$
f\left(\sigma_{i}\right)+\sum_{A \in P\left(\sigma_{i}\right)} s(A) f\left(\sigma_{i} \backslash A\right)=1 .
$$

Proof of Theorem 4.1: Let $\left(\sigma_{1}^{*}, \ldots, \sigma_{n}^{*}\right)$ be the combination of pure strategies constructed by the algorithm, and $h^{*}=\left(h_{e}^{*}\right)_{e \in E}$ the corresponding congestion vector. For all $1 \leq i \leq n$, the congestion vector $h^{*}$ satisfies the following two conditions:

$$
\begin{array}{cll}
\text { (i) } \quad W_{i}>l_{e}^{i}\left(h_{e}^{*}\right) & \forall e \in \sigma_{i}^{*} ; \\
\text { (ii) } W_{i} \leq l_{e}^{i}\left(h_{e}^{*}+1\right) & \forall e \in E \backslash \sigma_{i}^{*} .
\end{array}
$$

(14) is a result of the following observation. Choose any service provider $e$ and any agent $i$. If $e \in \sigma_{i}^{*}$, then $x_{e}^{i} \geq x_{e}^{\varphi_{e}^{-1}(k)}$, where

$$
k=\max _{j=1, \ldots, n}\left\{\varphi_{e}(j) \mid e \in \sigma_{j}^{*}\right\}=h_{e}^{*} .
$$

Since $e \in \sigma_{k}^{*}$, then $x_{e}^{\varphi_{e}^{-1}(k)} \geq k$, and $x_{e}^{i} \geq x_{e}^{\varphi_{e}^{-1}(k)} \geq k=h_{e}^{*}$. By the definition of $x_{e}^{i}$, for all $x \leq x_{e}^{i}$, we have $W_{i}>l_{e}^{i}(x)$. Thus, since $h_{e}^{*} \leq x_{e}^{i}$, we get $W_{i}>l_{e}^{i}\left(h_{e}^{*}\right)$.

If $e \notin \sigma_{i}^{*}$, then $x_{e}^{i} \leq x_{e}^{\varphi_{e}^{-1}(l)}$, where

$$
l=\min _{j=1, \ldots, n}\left\{\varphi_{e}(j) \mid e \notin \sigma_{j}^{*}\right\}=\max _{j=1, \ldots, n}\left\{\varphi_{e}(j) \mid e \in \sigma_{j}^{*}\right\}+1=h_{e}^{*}+1 .
$$

Since $e \notin \sigma_{l}^{*}$, then $x_{e}^{\varphi_{e}^{-1}(l)}<l$, and $x_{e}^{i} \leq x_{e}^{\varphi_{e}^{-1}(l)}<l=h_{e}^{*}+1$. By the definition of $x_{e}^{i}$, for all $x>x_{e}^{i}$, we have $W_{i} \leq l_{e}^{i}(x)$. Thus, since $h_{e}^{*}+1>x_{e}^{i}$, we get $W_{i} \leq l_{e}^{i}\left(h_{e}^{*}+1\right)$.

We show below that the combination of strategies $\left(\sigma_{1}^{*}, \ldots, \sigma_{n}^{*}\right)$ constructed by the algorithm is in equilibrium, i.e. for each agent $i$, for all $\sigma_{i} \in \Sigma_{i}, \pi_{i}\left(\sigma_{1}^{*}, \ldots, \sigma_{i}^{*}, \ldots, \sigma_{n}^{*}\right) \leq$ $\pi_{i}\left(\sigma_{1}^{*}, \ldots, \sigma_{i}, \ldots, \sigma_{n}^{*}\right)$. For all $\sigma_{i} \in \Sigma_{i}$, we denote $\pi_{i}\left(\sigma_{1}^{*}, \ldots, \sigma_{i}, \ldots, \sigma_{n}^{*}\right)$ by $\pi_{i}\left(\sigma_{i}\right)$. Thus, we want to show $\pi_{i}\left(\sigma_{i}^{*}\right) \leq \pi_{i}\left(\sigma_{i}\right)$, for all $i$ and $\sigma_{i}$.

We make a distinction between two possible cases: the strategy of agent $i$ is empty $\left(\sigma_{i}^{*}=\varnothing\right)$; the strategy of agent $i$ is nonempty $\left(\sigma_{i}^{*} \neq \varnothing\right)$. If agent $i$ chooses an empty set of SPs, i.e. $\sigma_{i}^{*}=\varnothing$, his disutility is $\pi_{i}\left(\sigma_{i}^{*}\right)=W_{i}$. The disutility of agent $i$ deviating from the empty strategy to any $\sigma_{i} \neq \varnothing$ is

$$
\begin{aligned}
\pi_{i}\left(\sigma_{i}\right) & =W_{i} f\left(\sigma_{i}\right)+\sum_{A \in P\left(\sigma_{i}\right)} l_{A}^{i}\left(h_{A}^{*}+1\right) s(A) f\left(\sigma_{i} \backslash A\right) \\
& \geq W_{i} f\left(\sigma_{i}\right)+W_{i} \sum_{A \in P\left(\sigma_{i}\right)} s(A) f\left(\sigma_{i} \backslash A\right)=W_{i}=\pi_{i}\left(\sigma_{i}^{*}\right) .
\end{aligned}
$$

The inequality follows from (14). Thus, for the first case we get $\pi_{i}\left(\sigma_{i}^{*}\right) \leq \pi_{i}\left(\sigma_{i}\right)$.

If agent $i$ chooses a nonempty strategy $\sigma_{i}^{*} \neq \varnothing$, his disutility is

$$
\begin{aligned}
\pi_{i}\left(\sigma_{i}^{*}\right) & =W_{i} f\left(\sigma_{i}^{*}\right)+\sum_{B \in P\left(\sigma_{i}^{*}\right)} l_{B}^{i}\left(h_{B}^{*}\right) s(B) f\left(\sigma_{i}^{*} \backslash B\right) \\
& <W_{i} f\left(\sigma_{i}^{*}\right)+W_{i} \sum_{B \in P\left(\sigma_{i}^{*}\right)} s(B) f\left(\sigma_{i}^{*} \backslash B\right)=W_{i}=\pi_{i}(\varnothing) .
\end{aligned}
$$


The inequality follows from (14). Thus, agent $i$ loses from the deviation to an empty strategy.

The disutility of agent $i$ deviating to any nonempty set of SPs $\sigma_{i} \neq \sigma_{i}^{*}$ is

$$
\pi_{i}\left(\sigma_{i}\right)=W_{i} f\left(\sigma_{i}\right)+\sum_{A \in P\left(\sigma_{i}\right)} l_{A}^{i}\left(h_{A}^{\sigma}\right) s(A) f\left(\sigma_{i} \backslash A\right),
$$

where

$$
h_{e}^{\sigma}= \begin{cases}h_{e}^{*} & e \in \sigma_{i}^{*} \cap \sigma_{i} ; \\ h_{e}^{*}+1 & e \in \sigma_{i} \backslash \sigma_{i}^{*} .\end{cases}
$$

We show below that

$$
\begin{aligned}
\pi_{i}\left(\sigma_{i}\right)-\pi_{i}\left(\sigma_{i}^{*}\right) & =W_{i} f\left(\sigma_{i}\right)+\sum_{A \in P\left(\sigma_{i}\right)} l_{A}^{i}\left(h_{A}^{\sigma}\right) s(A) f\left(\sigma_{i} \backslash A\right) \\
& -\left(W_{i} f\left(\sigma_{i}^{*}\right)+\sum_{B \in P\left(\sigma_{i}^{*}\right)} l_{B}^{i}\left(h_{B}^{*}\right) s(B) f\left(\sigma_{i}^{*} \backslash B\right)\right)=W_{i}\left(f\left(\sigma_{i}\right)-f\left(\sigma_{i}^{*}\right)\right) \\
& +\sum_{A \in P\left(\sigma_{i}\right)} l_{A}^{i}\left(h_{A}^{\sigma}\right) s(A) f\left(\sigma_{i} \backslash A\right)-\sum_{B \in P\left(\sigma_{i}^{*}\right)} l_{B}^{i}\left(h_{B}^{*}\right) s(B) f\left(\sigma_{i}^{*} \backslash B\right)
\end{aligned}
$$

is positive, i.e. $\pi_{i}\left(\sigma_{i}\right)>\pi_{i}\left(\sigma_{i}^{*}\right)$.

For every pair of sets $x, y$, the next equality holds:

$$
P(x)=P(x \cap y) \cup P(x \backslash y) \cup\{\Omega \cup \Psi \mid \Omega \in P(x \cap y), \Psi \in P(x \backslash y)\} .
$$

Then, by (18) and (20),

$$
\begin{aligned}
\pi_{i}\left(\sigma_{i}\right)-\pi_{i}\left(\sigma_{i}^{*}\right)= & W_{i}\left(f\left(\sigma_{i}\right)-f\left(\sigma_{i}^{*}\right)\right)+\left[\sum_{\Omega \in P\left(\sigma_{i} \cap \sigma_{i}^{*}\right)} l_{\Omega}^{i}\left(h_{\Omega}^{*}\right) s(\Omega) f\left(\sigma_{i} \backslash \Omega\right)\right. \\
& +\sum_{A^{\prime} \in P\left(\sigma_{i} \backslash \sigma_{i}^{*}\right)} l_{A^{\prime}}^{i}\left(h_{A^{\prime}}^{*}+1\right) s\left(A^{\prime}\right) f\left(\sigma_{i} \backslash A^{\prime}\right) \\
& \left.+\sum_{\Omega \in P\left(\sigma_{i} \cap \sigma_{i}^{*}\right)} \sum_{A^{\prime} \in P\left(\sigma_{i} \backslash \sigma_{i}^{*}\right)} l_{A^{\prime} \cup \Omega}^{i}\left(h_{A^{\prime} \cup \Omega}^{\sigma}\right) s\left(A^{\prime} \cup \Omega\right) f\left(\sigma_{i} \backslash\left(A^{\prime} \cup \Omega\right)\right)\right] \\
- & {\left[\sum_{\Omega \in P\left(\sigma_{i} \cap \sigma_{i}^{*}\right)} l_{\Omega}^{i}\left(h_{\Omega}^{*}\right) s(\Omega) f\left(\sigma_{i}^{*} \backslash \Omega\right)+\sum_{B^{\prime} \in P\left(\sigma_{i}^{*} \backslash \sigma_{i}\right)} l_{B^{\prime}}^{i}\left(h_{B^{\prime}}^{*}\right) s\left(B^{\prime}\right) f\left(\sigma_{i}^{*} \backslash B^{\prime}\right)\right.} \\
& \left.+\sum_{\Omega \in P\left(\sigma_{i} \cap \sigma_{i}^{*}\right)} \sum_{B^{\prime} \in P\left(\sigma_{i}^{*} \backslash \sigma_{i}\right)} l_{B^{\prime} \cup \Omega}^{i}\left(h_{B^{\prime} \cup \Omega}^{*}\right) s\left(B^{\prime} \cup \Omega\right) f\left(\sigma_{i}^{*} \backslash\left(B^{\prime} \cup \Omega\right)\right)\right] .
\end{aligned}
$$

For every pair of sets $x, y$ and for any $\Omega \in P(x \cap y), \Psi \in P(x \backslash y)$ the next equalities hold:

$$
\begin{aligned}
& x \backslash \Omega=((x \cap y) \backslash \Omega) \cup(x \backslash y) ; \\
& x \backslash(\Psi \cup \Omega)=((x \cap y) \backslash \Omega) \cup((x \backslash y) \backslash \Psi) .
\end{aligned}
$$


Using equalities (22), (23) and the fact that all sets $\Omega \in P\left(\sigma_{i} \cap \sigma_{i}^{*}\right), A^{\prime} \in P\left(\sigma_{i} \backslash \sigma_{i}^{*}\right)$, and $B^{\prime} \in P\left(\sigma_{i}^{*} \backslash \sigma_{i}\right)$ are disjoint, we get:

$$
\begin{aligned}
& \pi_{i}\left(\sigma_{i}\right)-\pi_{i}\left(\sigma_{i}^{*}\right)=W_{i}\left(f\left(\sigma_{i}\right)-f\left(\sigma_{i}^{*}\right)\right)+\sum_{A^{\prime} \in P\left(\sigma_{i} \backslash \sigma_{i}^{*}\right)} l_{A^{\prime}}^{i}\left(h_{A^{\prime}}^{*}+1\right) s\left(A^{\prime}\right) f\left(\sigma_{i} \backslash A^{\prime}\right) \\
& -\sum_{B^{\prime} \in P\left(\sigma_{i}^{*} \backslash \sigma_{i}\right)} l_{B^{\prime}}^{i}\left(h_{B^{\prime}}^{*}\right) s\left(B^{\prime}\right) f\left(\sigma_{i}^{*} \backslash B^{\prime}\right)+\left[\sum_{\Omega \in P\left(\sigma_{i} \cap \sigma_{i}^{*}\right)} l_{\Omega}^{i}\left(h_{\Omega}^{*}\right) s(\Omega) f\left(\left(\sigma_{i} \cap \sigma_{i}^{*}\right) \backslash \Omega\right) f\left(\sigma_{i} \backslash \sigma_{i}^{*}\right)\right. \\
& \left.+\sum_{\Omega \in P\left(\sigma_{i} \cap \sigma_{i}^{*}\right)} \sum_{A^{\prime} \in P\left(\sigma_{i} \backslash \sigma_{i}^{*}\right)} l_{A^{\prime} \cup \Omega}^{i}\left(h_{A^{\prime} \cup \Omega}^{\sigma}\right) s\left(A^{\prime}\right) s(\Omega) f\left(\left(\sigma_{i} \cap \sigma_{i}^{*}\right) \backslash \Omega\right) f\left(\left(\sigma_{i} \backslash \sigma_{i}^{*}\right) \backslash A^{\prime}\right)\right] \\
& -\left[\sum_{\Omega \in P\left(\sigma_{i} \cap \sigma_{i}^{*}\right)} l_{\Omega}^{i}\left(h_{\Omega}^{*}\right) s(\Omega) f\left(\left(\sigma_{i} \cap \sigma_{i}^{*}\right) \backslash \Omega\right) f\left(\sigma_{i}^{*} \backslash \sigma_{i}\right)\right. \\
& \left.+\sum_{\Omega \in P\left(\sigma_{i} \cap \sigma_{i}^{*}\right)} \sum_{B^{\prime} \in P\left(\sigma_{i}^{*} \backslash \sigma_{i}\right)} l_{B^{\prime} \cup \Omega}^{i}\left(h_{B^{\prime} \cup \Omega}^{*}\right) s\left(B^{\prime}\right) s(\Omega) f\left(\left(\sigma_{i} \cap \sigma_{i}^{*}\right) \backslash \Omega\right) f\left(\left(\sigma_{i}^{*} \backslash \sigma_{i}\right) \backslash B^{\prime}\right)\right] .
\end{aligned}
$$

For all $\Omega \in P\left(\sigma_{i} \cap \sigma_{i}^{*}\right), A^{\prime} \in P\left(\sigma_{i} \backslash \sigma_{i}^{*}\right)$, and $B^{\prime} \in P\left(\sigma_{i}^{*} \backslash \sigma_{i}\right)$, from (14) and (18) we get $l_{\Omega}^{i}\left(h_{\Omega}^{*}\right) \leq l_{A^{\prime} \cup \Omega}^{i}\left(h_{A^{\prime} \cup \Omega}\right)$, and since $\Omega \subseteq B^{\prime} \cup \Omega$, then $l_{B^{\prime} \cup \Omega}^{i}\left(h_{B^{\prime} \cup \Omega}^{*}\right) \leq l_{\Omega}^{i}\left(h_{\Omega}^{*}\right)$. Thus, for all $\Omega, A^{\prime}, B^{\prime}$, we have

$$
l_{B^{\prime} \cup \Omega}^{i}\left(h_{B^{\prime} \cup \Omega}^{*}\right) \leq l_{\Omega}^{i}\left(h_{\Omega}^{*}\right) \leq l_{A^{\prime} \cup \Omega}^{i}\left(h_{A^{\prime} \cup \Omega}^{\sigma}\right) .
$$

Using (25), we get

$$
\begin{aligned}
\pi_{i}\left(\sigma_{i}\right)-\pi_{i}\left(\sigma_{i}^{*}\right) \geq & W_{i}\left(f\left(\sigma_{i}\right)-f\left(\sigma_{i}^{*}\right)\right)+\sum_{A^{\prime} \in P\left(\sigma_{i} \backslash \sigma_{i}^{*}\right)} l_{A^{\prime}}^{i}\left(h_{A^{\prime}}^{*}+1\right) s\left(A^{\prime}\right) f\left(\sigma_{i} \backslash A^{\prime}\right) \\
- & \sum_{B^{\prime} \in P\left(\sigma_{i}^{*} \backslash \sigma_{i}\right)} l_{B^{\prime}}^{i}\left(h_{B^{\prime}}^{*}\right) s\left(B^{\prime}\right) f\left(\sigma_{i}^{*} \backslash B^{\prime}\right)+\sum_{\Omega \in P\left(\sigma_{i} \cap \sigma_{i}^{*}\right)} l_{\Omega}^{i}\left(h_{\Omega}^{*}\right) s(\Omega) f\left(\left(\sigma_{i} \cap \sigma_{i}^{*}\right) \backslash \Omega\right) \\
& \times\left[\sum_{A^{\prime} \in P\left(\sigma_{i} \backslash \sigma_{i}^{*}\right)} s\left(A^{\prime}\right) f\left(\left(\sigma_{i} \backslash \sigma_{i}^{*}\right) \backslash A^{\prime}\right)-\sum_{B^{\prime} \in P\left(\sigma_{i}^{*} \backslash \sigma_{i}\right)} s\left(B^{\prime}\right) f\left(\left(\sigma_{i}^{*} \backslash \sigma_{i}\right) \backslash B^{\prime}\right)\right. \\
& \left.+f\left(\sigma_{i} \backslash \sigma_{i}^{*}\right)-f\left(\sigma_{i}^{*} \backslash \sigma_{i}\right)\right] .
\end{aligned}
$$

By (13),

$$
\begin{aligned}
& \sum_{A^{\prime} \in P\left(\sigma_{i} \backslash \sigma_{i}^{*}\right)} s\left(A^{\prime}\right) f\left(\left(\sigma_{i} \backslash \sigma_{i}^{*}\right) \backslash A^{\prime}\right)-\sum_{B^{\prime} \in P\left(\sigma_{i}^{*} \backslash \sigma_{i}\right)} s\left(B^{\prime}\right) f\left(\left(\sigma_{i}^{*} \backslash \sigma_{i}\right) \backslash B^{\prime}\right) \\
& \quad+f\left(\sigma_{i} \backslash \sigma_{i}^{*}\right)-f\left(\sigma_{i}^{*} \backslash \sigma_{i}\right)=\left(1-f\left(\sigma_{i} \backslash \sigma_{i}^{*}\right)\right)-\left(1-f\left(\sigma_{i}^{*} \backslash \sigma_{i}\right)\right) \\
& +f\left(\sigma_{i} \backslash \sigma_{i}^{*}\right)-f\left(\sigma_{i}^{*} \backslash \sigma_{i}\right)=0 .
\end{aligned}
$$


By (13), (20), (22), (23), (27),

$$
\begin{aligned}
f\left(\sigma_{i}\right)-f\left(\sigma_{i}^{*}\right)= & \sum_{B^{\prime} \in P\left(\sigma_{i}^{*} \backslash \sigma_{i}\right)} s\left(B^{\prime}\right) f\left(\sigma_{i}^{*} \backslash B^{\prime}\right)-\sum_{A^{\prime} \in P\left(\sigma_{i} \backslash \sigma_{i}^{*}\right)} s\left(A^{\prime}\right) f\left(\sigma_{i} \backslash A^{\prime}\right) \\
+ & \sum_{\Omega \in P\left(\sigma_{i} \cap \sigma_{i}^{*}\right)} s(\Omega) f\left(\left(\sigma_{i} \cap \sigma_{i}^{*}\right) \backslash \Omega\right) \times\left[\sum_{B^{\prime} \in P\left(\sigma_{i}^{*} \backslash \sigma_{i}\right)} s\left(B^{\prime}\right) f\left(\left(\sigma_{i}^{*} \backslash \sigma_{i}\right) \backslash B^{\prime}\right)\right. \\
& \left.-\sum_{A^{\prime} \in P\left(\sigma_{i} \backslash \sigma_{i}^{*}\right)} s\left(A^{\prime}\right) f\left(\left(\sigma_{i} \backslash \sigma_{i}^{*}\right) \backslash A^{\prime}\right)+f\left(\sigma_{i}^{*} \backslash \sigma_{i}\right)-f\left(\sigma_{i} \backslash \sigma_{i}^{*}\right)\right] \\
= & \sum_{B^{\prime} \in P\left(\sigma_{i}^{*} \backslash \sigma_{i}\right)} s\left(B^{\prime}\right) f\left(\sigma_{i}^{*} \backslash B^{\prime}\right)-\sum_{A^{\prime} \in P\left(\sigma_{i} \backslash \sigma_{i}^{*}\right)} s\left(A^{\prime}\right) f\left(\sigma_{i} \backslash A^{\prime}\right) .
\end{aligned}
$$

Inserting (27) and (28) into (26), we get

$$
\begin{aligned}
& \pi_{i}\left(\sigma_{i}\right)-\pi_{i}\left(\sigma_{i}^{*}\right) \geq W_{i} \sum_{B^{\prime} \in P\left(\sigma_{i}^{*} \backslash \sigma_{i}\right)} s\left(B^{\prime}\right) f\left(\sigma_{i}^{*} \backslash B^{\prime}\right)-\sum_{B^{\prime} \in P\left(\sigma_{i}^{*} \backslash \sigma_{i}\right)} l_{B^{\prime}}^{i}\left(h_{B^{\prime}}^{*}\right) s\left(B^{\prime}\right) f\left(\sigma_{i}^{*} \backslash B^{\prime}\right) \\
& +\sum_{A^{\prime} \in P\left(\sigma_{i} \backslash \sigma_{i}^{*}\right)} l_{A^{\prime}}^{i}\left(h_{A^{\prime}}^{*}+1\right) s\left(A^{\prime}\right) f\left(\sigma_{i} \backslash A^{\prime}\right)-W_{i} \sum_{A^{\prime} \in P\left(\sigma_{i} \backslash \sigma_{i}^{*}\right)} s\left(A^{\prime}\right) f\left(\sigma_{i} \backslash A^{\prime}\right) .
\end{aligned}
$$

By $(14), W_{i}>l_{B^{\prime}}^{i}\left(h_{B^{\prime}}^{*}\right)$ for all $B^{\prime} \in P\left(\sigma_{i}^{*} \backslash \sigma_{i}\right)$, and $W_{i} \leq l_{A^{\prime}}^{i}\left(h_{A^{\prime}}^{*}+1\right)$ for all $A^{\prime} \in P\left(\sigma_{i} \backslash \sigma_{i}^{*}\right)$. Thus $\pi_{i}\left(\sigma_{i}\right)-\pi_{i}\left(\sigma_{i}^{*}\right)>0$.

Therefore, $\pi_{i}\left(\sigma_{i}^{*}\right) \leq \pi_{i}\left(\sigma_{i}\right)$, for all $\sigma_{i}^{*}, \sigma_{i} \in \Sigma_{i}$, as claimed.

\section{Proof of Claim 4.3:}

(i) Suppose there is an agent $i$ such that the set $L_{i} \subseteq \sigma_{i}$ of SPs with $l_{e}^{i}\left(h_{e}^{\sigma}\right)>W_{i}$ is not empty: $L_{i}=\left\{e \in \sigma_{i} \mid l_{e}^{i}\left(h_{e}^{\sigma}\right)>W_{i}\right\} \neq \varnothing$. Let $b \in L_{i}$ be such SP with maximal $l_{e}^{i}\left(h_{e}^{\sigma}\right)$ : $b \in \arg \max _{e \in L_{i}} l_{e}^{i}\left(h_{e}^{\sigma}\right)$.

We show below that $\pi_{i}(\sigma)>\pi_{i}\left(\sigma_{1}, \ldots, \sigma_{i} \backslash\{b\}, \ldots, \sigma_{n}\right)$, i.e. $\sigma$ is not a Nash equilibrium strategy profile, in contradiction.

We denote $\left(\sigma_{1}, \ldots, \sigma_{i} \backslash\{b\}, \ldots, \sigma_{n}\right)$ by $\hat{\sigma}$.

$$
\begin{aligned}
\pi_{i}(\sigma)-\pi_{i}(\hat{\sigma})= & W_{i} f\left(\sigma_{i}\right)+\sum_{A \in P\left(\sigma_{i}\right)} l_{A}^{i}\left(h_{A}^{\sigma}\right) s(A) f\left(\sigma_{i} \backslash A\right) \\
& -W_{i} f\left(\sigma_{i} \backslash\{b\}\right)-\sum_{B \in P\left(\sigma_{i} \backslash\{b\}\right)} l_{B}^{i}\left(h_{B}^{\hat{\sigma}}\right) s(B) f\left(\left(\sigma_{i} \backslash\{b\}\right) \backslash B\right) .
\end{aligned}
$$

By (20),

$$
\begin{aligned}
\pi_{i}(\sigma)-\pi_{i}(\hat{\sigma})= & W_{i} f\left(\sigma_{i} \backslash\{b\}\right)\left(f_{b}-1\right)+\sum_{B \in P\left(\sigma_{i} \backslash\{b\}\right)} l_{B}^{i}\left(h_{B}^{\sigma}\right) s(B) f\left(\sigma_{i} \backslash B\right) \\
& -\sum_{B \in P\left(\sigma_{i} \backslash\{b\}\right)} l_{B}^{i}\left(h_{B}^{\sigma}\right) s(B) f\left(\left(\sigma_{i} \backslash\{b\}\right) \backslash B\right)+l_{b}^{i}\left(h_{b}^{\sigma}\right) s_{b} f\left(\sigma_{i} \backslash\{b\}\right) \\
& +\sum_{B \in P\left(\sigma_{i} \backslash\{b\}\right)} l_{B \cup\{b\}}^{i}\left(h_{B \cup\{b\}}^{\sigma}\right) s_{b} s(B) f\left(\left(\sigma_{i} \backslash(B \cup\{b\})\right) .\right.
\end{aligned}
$$


Since $\sigma_{i} \backslash(B \cup\{b\})=\left(\sigma_{i} \backslash\{b\}\right) \backslash B$ and $\sigma_{i} \backslash B=\left(\left(\sigma_{i} \backslash\{b\}\right) \backslash B\right) \cup\{b\}$,

$$
\begin{aligned}
\pi_{i}(\sigma)-\pi_{i}(\hat{\sigma})= & s_{b}\left[f\left(\sigma_{i} \backslash\{b\}\right)\left(l_{b}^{i}\left(h_{b}^{\sigma}\right)-W_{i}\right)\right. \\
& \left.+\sum_{B \in P\left(\sigma_{i} \backslash\{b\}\right)} s(B) f\left(\left(\sigma_{i} \backslash\{b\}\right) \backslash B\right)\left(l_{B \cup\{b\}}^{i}\left(h_{B \cup\{b\}}^{\sigma}\right)-l_{B}^{i}\left(h_{B}^{\sigma}\right)\right)\right] .
\end{aligned}
$$

If for all $B \in P\left(\sigma_{i} \backslash\{b\}\right), l_{b}^{i}\left(h_{b}^{\sigma}\right) \geq l_{B}^{i}\left(h_{B}^{\sigma}\right)$, then $l_{B \cup\{b\}}^{i}\left(h_{B \cup\{b\}}^{\sigma}\right)=l_{B}^{i}\left(h_{B}^{\sigma}\right)$, and we are done. Else, there is $B \in P\left(\sigma_{i} \backslash\{b\}\right)$ such that $l_{b}^{i}\left(h_{b}^{\sigma}\right)<l_{B}^{i}\left(h_{B}^{\sigma}\right)$, i.e. $l_{e}^{i}\left(h_{e}^{\sigma}\right)>$ $l_{b}^{i}\left(h_{b}^{\sigma}\right)>W_{i}$ for all $e \in B$. Therefore, there exists $c \in L_{i}$ such that $l_{c}^{i}\left(h_{c}^{\sigma}\right)>l_{b}^{i}\left(h_{b}^{\sigma}\right)$, in contradiction to the choice of $b$.

(ii) Suppose there are agent $i$ and SP $b \in E$ such that $l_{b}^{i}\left(h_{b}^{\sigma}+1\right)<W_{i}$ and $b \notin \sigma_{i}$. We show below that $\pi_{i}(\sigma)>\pi_{i}\left(\sigma_{1}, \ldots, \sigma_{i} \cup\{b\}, \ldots, \sigma_{n}\right)$, i.e. $\sigma$ is not a Nash equilibrium strategy profile, in contradiction.

We denote $\left(\sigma_{1}, \ldots, \sigma_{i} \cup\{b\}, \ldots, \sigma_{n}\right)$ by $\hat{\sigma}$.

$$
\begin{aligned}
\pi_{i}(\sigma)-\pi_{i}(\hat{\sigma})= & W_{i} f\left(\sigma_{i}\right)+\sum_{A \in P\left(\sigma_{i}\right)} l_{A}^{i}\left(h_{A}^{\sigma}\right) s(A) f\left(\sigma_{i} \backslash A\right) \\
& -W_{i} f\left(\sigma_{i} \cup\{b\}\right)-\sum_{B \in P\left(\sigma_{i} \cup\{b\}\right)} l_{B}^{i}\left(h_{B}^{\hat{\sigma}}\right) s(B) f\left(\left(\sigma_{i} \cup\{b\}\right) \backslash B\right) .
\end{aligned}
$$

By $(20)$,

$$
\begin{aligned}
\pi_{i}(\sigma)-\pi_{i}(\hat{\sigma})= & W_{i} f\left(\sigma_{i}\right)\left(1-f_{b}\right)+\sum_{A \in P\left(\sigma_{i}\right)} l_{A}^{i}\left(h_{A}^{\sigma}\right) s(A) f\left(\sigma_{i} \backslash A\right) \\
& -\sum_{A \in P\left(\sigma_{i}\right)} l_{A}^{i}\left(h_{A}^{\sigma}\right) s(A) f\left(\left(\sigma_{i} \cup\{b\}\right) \backslash A\right)-l_{b}^{i}\left(h_{b}^{\sigma}+1\right) s_{b} f\left(\sigma_{i}\right) \\
& -\sum_{A \in P\left(\sigma_{i}\right)} l_{A \cup\{b\}}^{i}\left(h_{A \cup\{b\}}^{\hat{\sigma}}\right) s_{b} s(A) f\left(\left(\sigma_{i} \cup\{b\}\right) \backslash(A \cup\{b\})\right) .
\end{aligned}
$$

Since $\left(\sigma_{i} \cup\{b\}\right) \backslash(A \cup\{b\})=\sigma_{i} \backslash A$ and $\left(\sigma_{i} \cup\{b\}\right) \backslash A=\left(\sigma_{i} \backslash A\right) \cup\{b\}$,

$$
\begin{aligned}
\pi_{i}(\sigma)-\pi_{i}(\hat{\sigma})= & s_{b}\left[f\left(\sigma_{i}\right)\left(W_{i}-l_{b}^{i}\left(h_{b}^{\sigma}+1\right)\right)\right. \\
& \left.+\sum_{A \in P\left(\sigma_{i}\right)} s(A) f\left(\sigma_{i} \backslash A\right)\left(l_{A}^{i}\left(h_{A}^{\sigma}\right)-l_{A \cup\{b\}}^{i}\left(h_{A \cup\{b\}}^{\hat{\sigma}}\right)\right)\right] .
\end{aligned}
$$

For all $A \in P\left(\sigma_{i}\right), l_{A}^{i}\left(h_{A}^{\sigma}\right) \geq l_{A \cup\{b\}}^{i}\left(h_{A \cup\{b\}}^{\hat{\sigma}}\right)$ (because $A \subset(A \cup\{b\})$ and $h_{e}^{\hat{\sigma}}=h_{e}^{\sigma}$, $\forall e \in E)$. Then, since $W_{i}>l_{b}^{i}\left(h_{b}^{\sigma}+1\right)$ and $f_{e}, s_{e}>0$ for all $e \in E$, we get $\pi_{i}(\sigma)-\pi_{i}(\hat{\sigma})>0$, i.e. $\pi_{i}(\sigma)>\pi_{i}(\hat{\sigma})$ and $\sigma \notin N E$, in contradiction.

Proof of Proposition 5.1: Let $\sigma \in N E$ be a combination of strategies at Nash equilibrium. Suppose there are two SPs $a, b \in E$ such that $h_{a}^{\sigma}>h_{b}^{\sigma}+1$. Then, there exists agent 
$i$ such that $a \in \sigma_{i}$, but $b \notin \sigma_{i}$. We show below that if agent $i$ deviates from $\sigma_{i}$ to new strategy $\sigma_{i}^{\prime}=\left(\sigma_{i} \backslash\{a\}\right) \cup\{b\}$, then his new disutility $\pi_{i}\left(\sigma^{\prime}\right)$, where $\sigma^{\prime}=\left(\sigma_{1}, \ldots, \sigma_{i}^{\prime}, \ldots, \sigma_{n}\right)$, is less than the disutility $\pi_{i}(\sigma)$, which implies that $\sigma$ is not an equilibrium strategy, in contradiction.

Since $\sigma_{i}$ is nonempty, the disutility of agent $i$ for this combination of strategies is

$$
\pi_{i}(\sigma)=W_{i} f\left(\sigma_{i}\right)+\sum_{A \in P\left(\sigma_{i}\right)} l_{A}\left(h_{A}^{\sigma}\right) s(A) f\left(\sigma_{i} \backslash A\right) .
$$

The disutility of agent $i$ for the new combination of strategies $\sigma^{\prime}$ is

$$
\pi_{i}\left(\sigma^{\prime}\right)=W_{i} f\left(\sigma_{i}^{\prime}\right)+\sum_{B \in P\left(\sigma_{i}^{\prime}\right)} l_{B}\left(h_{B}^{\sigma^{\prime}}\right) s(B) f\left(\sigma_{i} \backslash B\right),
$$

where

$$
h_{e}^{\sigma^{\prime}}= \begin{cases}h_{e}^{\sigma} & e \in \sigma_{i} \cap \sigma_{i}^{\prime} \\ h_{e}^{\sigma}+1 & e \in \sigma_{i}^{\prime} \backslash \sigma_{i} .\end{cases}
$$

We show below that $\pi_{i}(\sigma)-\pi_{i}\left(\sigma^{\prime}\right)=W_{i}\left(f\left(\sigma_{i}\right)-f\left(\sigma_{i}^{\prime}\right)\right)+\sum_{A \in P\left(\sigma_{i}\right)} l_{A}\left(h_{A}^{\sigma}\right) s(A) f\left(\sigma_{i} \backslash A\right)$

$$
-\sum_{B \in P\left(\sigma_{i}^{\prime}\right)} l_{B}\left(h_{B}^{\sigma^{\prime}}\right) s(B) f\left(\sigma_{i} \backslash B\right)
$$

is positive, i.e. $\pi_{i}(\sigma)>\pi_{i}\left(\sigma^{\prime}\right)$.

By (38) and (20),

$$
\begin{aligned}
& \pi_{i}(\sigma)-\pi_{i}\left(\sigma^{\prime}\right)=W_{i}\left(f\left(\sigma_{i}\right)-f\left(\sigma_{i}^{\prime}\right)\right)+\left[\sum_{\Omega \in P\left(\sigma_{i} \cap \sigma_{i}^{\prime}\right)} l_{\Omega}\left(h_{\Omega}^{\sigma}\right) s(\Omega) f\left(\sigma_{i} \backslash \Omega\right)\right. \\
& +\sum_{A^{\prime} \in P\left(\sigma_{i} \backslash \sigma_{i}^{\prime}\right)} l_{A^{\prime}}\left(h_{A^{\prime}}^{\sigma}\right) s\left(A^{\prime}\right) f\left(\sigma_{i} \backslash A^{\prime}\right) \\
& \left.+\sum_{\Omega \in P\left(\sigma_{i} \cap \sigma_{i}^{\prime}\right)} \sum_{A^{\prime} \in P\left(\sigma_{i} \backslash \sigma_{i}^{\prime}\right)} l_{A^{\prime} \cup \Omega}\left(h_{A^{\prime} \cup \Omega}^{\sigma}\right) s\left(A^{\prime} \cup \Omega\right) f\left(\sigma_{i} \backslash\left(A^{\prime} \cup \Omega\right)\right)\right] \\
& -\left[\sum_{\Omega \in P\left(\sigma_{i} \cap \sigma_{i}^{\prime}\right)} l_{\Omega}^{i}\left(h_{\Omega}^{\sigma}\right) s(\Omega) f\left(\sigma_{i}^{\prime} \backslash \Omega\right)+\sum_{B^{\prime} \in P\left(\sigma_{i}^{\prime} \backslash \sigma_{i}\right)} l_{B^{\prime}}\left(h_{B^{\prime}}^{\sigma}+1\right) s\left(B^{\prime}\right) f\left(\sigma_{i}^{\prime} \backslash B^{\prime}\right)\right. \\
& \left.+\sum_{\Omega \in P\left(\sigma_{i} \cap \sigma_{i}^{\prime}\right)} \sum_{B^{\prime} \in P\left(\sigma_{i}^{\prime} \backslash \sigma_{i}\right)} l_{B^{\prime} \cup \Omega}\left(h_{B^{\prime} \cup \Omega}^{\sigma^{\prime}}\right) s\left(B^{\prime} \cup \Omega\right) f\left(\sigma_{i}^{\prime} \backslash\left(B^{\prime} \cup \Omega\right)\right)\right] \text {. }
\end{aligned}
$$

Using equality (22) and the fact that all sets $\Omega \in P\left(\sigma_{i} \cap \sigma_{i}^{\prime}\right), A^{\prime} \in P\left(\sigma_{i} \backslash \sigma_{i}^{\prime}\right)$, and $B^{\prime} \in P\left(\sigma_{i}^{\prime} \backslash \sigma_{i}\right)$ are disjoint, we get: 


$$
\begin{aligned}
\pi_{i}(\sigma)-\pi_{i}\left(\sigma^{\prime}\right) & =W_{i} f\left(\sigma_{i} \cap \sigma_{i}^{\prime}\right)\left[f\left(\sigma_{i} \backslash \sigma_{i}^{\prime}\right)-f\left(\sigma_{i}^{\prime} \backslash \sigma_{i}\right)\right]+\sum_{A^{\prime} \in P\left(\sigma_{i} \backslash \sigma_{i}^{\prime}\right)} l_{A^{\prime}}\left(h_{A^{\prime}}^{\sigma}\right) s\left(A^{\prime}\right) f\left(\sigma_{i} \backslash A^{\prime}\right) \\
& -\sum_{B^{\prime} \in P\left(\sigma_{i}^{\prime} \backslash \sigma_{i}\right)} l_{B^{\prime}}\left(h_{B^{\prime}}^{\sigma}+1\right) s\left(B^{\prime}\right) f\left(\sigma_{i}^{\prime} \backslash B^{\prime}\right) \\
& +\left[\sum_{\Omega \in P\left(\sigma_{i} \cap \sigma_{i}^{\prime}\right)} l_{\Omega}\left(h_{\Omega}^{\sigma}\right) s(\Omega) f\left(\left(\sigma_{i} \cap \sigma_{i}^{\prime}\right) \backslash \Omega\right) f\left(\sigma_{i} \backslash \sigma_{i}^{\prime}\right)\right. \\
& \left.+\sum_{\Omega \in P\left(\sigma_{i} \cap \sigma_{i}^{\prime}\right)} \sum_{A^{\prime} \in P\left(\sigma_{i} \backslash \sigma_{i}^{\prime}\right)} l_{A^{\prime} \cup \Omega}\left(h_{A^{\prime} \cup \Omega}^{\sigma}\right) s\left(A^{\prime}\right) s(\Omega) f\left(\sigma_{i} \backslash\left(A^{\prime} \cup \Omega\right)\right)\right] \\
& -\left[\sum_{\Omega \in P\left(\sigma_{i} \cap \sigma_{i}^{\prime}\right)} l_{\Omega}\left(h_{\Omega}^{\sigma}\right) s(\Omega) f\left(\left(\sigma_{i} \cap \sigma_{i}^{\prime}\right) \backslash \Omega\right) f\left(\sigma_{i}^{\prime} \backslash \sigma_{i}\right)\right. \\
& \left.+\sum_{\Omega \in P\left(\sigma_{i} \cap \sigma_{i}^{\prime}\right) B^{\prime} \in P\left(\sigma_{i}^{\prime} \backslash \sigma_{i}\right)} l_{B^{\prime} \cup \Omega}\left(h_{B^{\prime} \cup \Omega}^{\sigma^{\prime}}\right) s\left(B^{\prime}\right) s(\Omega) f\left(\sigma_{i}^{\prime} \backslash\left(B^{\prime} \cup \Omega\right)\right)\right]
\end{aligned}
$$

Since $\sigma_{i}^{\prime}=\left(\sigma_{i} \backslash\{a\}\right) \cup\{b\}$, then

$$
\begin{aligned}
\sigma_{i} \cap \sigma_{i}^{\prime} & =\sigma_{i} \backslash\{a\}=\sigma_{i}^{\prime} \backslash\{b\} ; \\
\sigma_{i} \backslash \sigma_{i}^{\prime} & =\{a\} ; \\
\sigma_{i}^{\prime} \backslash \sigma_{i} & =\{b\} .
\end{aligned}
$$

For all sets $x, y, z$ the next equality holds:

$$
x \backslash(y \cup z)=(x \backslash y) \backslash z .
$$

By (42), (43) we get

$$
\begin{aligned}
\pi_{i}(\sigma)-\pi_{i}\left(\sigma^{\prime}\right) & =W_{i} f\left(\sigma_{i} \cap \sigma_{i}^{\prime}\right)\left(f_{a}-f_{b}\right)+l\left(h_{a}^{\sigma}\right) s_{a} f\left(\sigma_{i} \backslash\{a\}\right)-l\left(h_{b}^{\sigma}+1\right) s_{b} f\left(\sigma_{i} \backslash\{a\}\right) \\
& +\left(f_{a}-f_{b}\right) \sum_{\Omega \in P\left(\sigma_{i} \cap \sigma_{i}^{\prime}\right)} l_{\Omega}\left(h_{\Omega}^{\sigma}\right) s(\Omega) f\left(\left(\sigma_{i} \backslash\{a\}\right) \backslash \Omega\right) \\
& +\sum_{\Omega \in P\left(\sigma_{i} \cap \sigma_{i}^{\prime}\right)} l_{\{a\} \cup \Omega}\left(h_{\{a\} \cup \Omega}^{\sigma}\right) s_{a} s(\Omega) f\left(\left(\sigma_{i} \backslash\{a\}\right) \backslash \Omega\right) \\
& -\sum_{\Omega \in P\left(\sigma_{i} \cap \sigma_{i}^{\prime}\right)} l_{\{b\} \cup \Omega}\left(h_{\{b\} \cup \Omega}^{\sigma^{\prime}}\right) s_{b} s(\Omega) f\left(\left(\sigma_{i} \backslash\{a\}\right) \backslash \Omega\right) .
\end{aligned}
$$

Since all the SPs have the same failure and success probabilities,

$$
\begin{aligned}
\pi_{i}(\sigma)-\pi_{i}\left(\sigma^{\prime}\right) & =s f\left(\sigma_{i} \backslash\{a\}\right)\left[l\left(h_{a}^{\sigma}\right)-l\left(h_{b}^{\sigma}+1\right)\right] \\
& +\sum_{\Omega \in P\left(\sigma_{i} \cap \sigma_{i}^{\prime}\right)} l_{\{a\} \cup \Omega}\left(h_{\{a\} \cup \Omega}^{\sigma}\right) s(\Omega) s f\left(\left(\sigma_{i} \backslash\{a\}\right) \backslash \Omega\right) \\
& -\sum_{\Omega \in P\left(\sigma_{i} \cap \sigma_{i}^{\prime}\right)} l_{\{b\} \cup \Omega}\left(h_{\{b\} \cup \Omega}^{\sigma^{\prime}}\right) s(\Omega) s f\left(\left(\sigma_{i} \backslash\{a\}\right) \backslash \Omega\right) .
\end{aligned}
$$

Because of the monotonicity of the function $l(x)$ on the interval $0 \leq x \leq h_{M}$, where $h_{M}=\max \left\{h_{e}^{\sigma} \mid e \in E, \sigma \in N E\right\}$, and since $h_{a}^{\sigma}>h_{b}^{\sigma}+1$, we get $l\left(h_{a}^{\sigma}\right)>l\left(h_{b}^{\sigma}+1\right)=l\left(h_{b}^{\sigma^{\prime}}\right)$. Then, for any $\Omega \in \sigma_{i} \cap \sigma_{i}^{\prime}$ we have

$$
l_{\{a\} \cup \Omega}\left(h_{\{a\} \cup \Omega}^{\sigma}\right) \geq l_{\{b\} \cup \Omega}\left(h_{\{b\} \cup \Omega}^{\sigma^{\prime}}\right) .
$$


Therefore, $\pi(\sigma)>\pi\left(\sigma^{\prime}\right)$, as claimed.

Proof of Claim 5.3: Since $\left|\sigma_{i}\right|>\left|\sigma_{j}\right|+1$, then $\sigma_{i} \backslash \sigma_{j}$ is not empty, and let $b \in \sigma_{i} \backslash \sigma_{j}$ be such a SP with maximal cost:

$$
b \in \arg \max _{e \in \sigma_{i} \backslash \sigma_{j}} l\left(h_{e}^{\sigma}\right) .
$$

We want to show that if we take this SP from agent $i$ and give it to agent $j$, the total disutility of the agents can only to be improved.

Obviously, for all $e \in E, h_{e}^{\hat{\sigma}}=h_{e}^{\sigma}$ (we will denote it by $h_{e}$ ). Then, since $\hat{\sigma_{k}}=\sigma_{k}$ for all $k \neq i, j$, we have $\pi_{k}(\hat{\sigma})=\pi_{k}(\sigma)$, for all $k \neq i, j$. Therefore, it suffices to show that

$$
\begin{gathered}
\pi_{i}(\hat{\sigma})+\pi_{j}(\hat{\sigma}) \leq \pi_{i}(\sigma)+\pi_{j}(\sigma) . \\
{\left[\pi_{i}(\sigma)+\pi_{j}(\sigma)\right]-\quad\left[\pi_{i}(\hat{\sigma})+\pi_{j}(\hat{\sigma})\right]=W f\left(\sigma_{i}\right)+\sum_{A^{\prime} \in P\left(\sigma_{i}\right)} l_{A^{\prime}}\left(h_{A^{\prime}}\right) s\left(A^{\prime}\right) f\left(\sigma_{i} \backslash A^{\prime}\right)} \\
+W f\left(\sigma_{j}\right)+\sum_{B \in P\left(\sigma_{j}\right)} l_{B}\left(h_{B}\right) s(B) f\left(\sigma_{j} \backslash B\right) \\
-W f\left(\sigma_{i} \backslash\{b\}\right)-\sum_{A \in P\left(\sigma_{i} \backslash\{b\}\right)} l_{A}\left(h_{A}\right) s(A) f\left(\left(\sigma_{i} \backslash\{b\}\right) \backslash A\right) \\
-W f\left(\sigma_{j} \cup\{b\}\right)-\sum_{B^{\prime} \in P\left(\sigma_{j} \cup\{b\}\right)} l_{B^{\prime}}\left(h_{B^{\prime}}\right) s\left(B^{\prime}\right) f\left(\left(\sigma_{j} \cup\{b\}\right) \backslash B^{\prime}\right) .
\end{gathered}
$$

By (20), and since $b \in \sigma_{i} \backslash \sigma_{j}$,

$$
\begin{gathered}
P\left(\sigma_{i}\right)=P\left(\sigma_{i} \backslash\{b\}\right) \cup\{b\} \cup\left\{A \cup\{b\} \mid A \in P\left(\sigma_{i} \backslash\{b\}\right)\right\} ; \\
P\left(\sigma_{j} \cup\{b\}\right)=P\left(\sigma_{j}\right) \cup\{b\} \cup\left\{B \cup\{b\} \mid B \in P\left(\sigma_{j}\right)\right\} .
\end{gathered}
$$

Then, by (48) and (49),

$$
\begin{aligned}
{\left[\pi_{i}(\sigma)+\pi_{j}(\sigma)\right]-} & {\left[\pi_{i}(\hat{\sigma})+\pi_{j}(\hat{\sigma})\right]=W f\left(\sigma_{i} \backslash\{b\}\right)\left(f_{b}-1\right)+W f\left(\sigma_{j}\right)\left(1-f_{b}\right) } \\
& +\sum_{A \in P\left(\sigma_{i} \backslash\{b\}\right)} l_{A}\left(h_{A}\right) s(A) f\left(\sigma_{i} \backslash A\right)+l\left(h_{b}\right) s_{b} f\left(\sigma_{i} \backslash\{b\}\right) \\
& +\sum_{A \in P\left(\sigma_{i} \backslash\{b\}\right)} l_{A \cup\{b\}}\left(h_{A \cup\{b\}}\right) s_{b} s(A) f\left(\sigma_{i} \backslash(A \cup\{b\})\right) \\
& +\sum_{B \in P\left(\sigma_{j}\right)} l_{B}\left(h_{B}\right) s(B) f\left(\sigma_{j} \backslash B\right)-\sum_{A \in P\left(\sigma_{i} \backslash\{b\}\right)} l_{A}\left(h_{A}\right) s(A) f\left(\left(\sigma_{i} \backslash\{b\}\right) \backslash A\right) \\
& -\sum_{B \in P\left(\sigma_{j}\right)} l_{B}\left(h_{B}\right) s(B) f\left(\left(\sigma_{j} \cup\{b\}\right) \backslash B\right)-l\left(h_{b}\right) s_{b} f\left(\sigma_{j}\right) \\
& -\sum_{B \in P\left(\sigma_{j}\right)} l_{B \cup\{b\}}\left(h_{B \cup\{b\}}\right) s_{b} s(B) f\left(\sigma_{j} \backslash B\right) .
\end{aligned}
$$


Simplifying this expression, we get

$$
\begin{aligned}
{\left[\pi_{i}(\sigma)+\pi_{j}(\sigma)\right] } & -\left[\pi_{i}(\hat{\sigma})+\pi_{j}(\hat{\sigma})\right]=s_{b}\left[\left(f\left(\sigma_{j}\right)-f\left(\sigma_{i} \backslash\{b\}\right)\right)\left(W-l\left(h_{b}\right)\right)\right. \\
& +\sum_{A \in P\left(\sigma_{i} \backslash\{b\}\right)} s(A) f\left(\left(\sigma_{i} \backslash\{b\}\right) \backslash A\right)\left(l_{A \cup\{b\}}\left(h_{A \cup\{b\}}\right)-l_{A}\left(h_{A}\right)\right) \\
& \left.+\sum_{B \in P\left(\sigma_{j}\right)} s(B) f\left(\sigma_{j} \backslash B\right)\left(l_{B}\left(h_{B}\right)-l_{B \cup\{b\}}\left(h_{B \cup\{b\}}\right)\right)\right] .
\end{aligned}
$$

By (20), and since $b \in \sigma_{i} \backslash \sigma_{j}$,

$$
\begin{aligned}
& P\left(\sigma_{i} \backslash\{b\}\right)= P\left(\sigma_{i} \cap \sigma_{j}\right) \cup P\left(\left(\sigma_{i} \backslash\{b\}\right) \backslash \sigma_{j}\right) \\
& \cup\left\{\Omega \cup A^{\prime} \mid \Omega \in P\left(\sigma_{i} \cap \sigma_{j}\right), A^{\prime} \in P\left(\left(\sigma_{i} \backslash\{b\}\right) \backslash \sigma_{j}\right)\right\} ; \\
& P\left(\sigma_{j}\right)= P\left(\sigma_{i} \cap \sigma_{j}\right) \cup P\left(\sigma_{j} \backslash\left(\sigma_{i} \backslash\{b\}\right)\right) \\
& \cup\left\{\Omega \cup B^{\prime} \mid \Omega \in P\left(\sigma_{i} \cap \sigma_{j}\right), B^{\prime} \in P\left(\sigma_{j} \backslash\left(\sigma_{i} \backslash\{b\}\right)\right)\right\} .
\end{aligned}
$$

Then, by (52) and (53),

$$
\begin{aligned}
{\left[\pi_{i}(\sigma)+\pi_{j}(\sigma)\right] } & -\left[\pi_{i}(\hat{\sigma})+\pi_{j}(\hat{\sigma})\right]=s_{b}\left[\left(f\left(\sigma_{j}\right)-f\left(\sigma_{i} \backslash\{b\}\right)\right)\left(W-l\left(h_{b}\right)\right)\right. \\
& +\sum_{\Omega \in P\left(\sigma_{i} \cap \sigma_{j}\right)}\left(l_{\Omega}\left(h_{\Omega}\right)-l_{\Omega \cup\{b\}}\left(h_{\Omega \cup\{b\}}\right)\right) s(\Omega)\left(f\left(\sigma_{j} \backslash \Omega\right)-f\left(\left(\sigma_{i} \backslash\{b\}\right) \backslash \Omega\right)\right) \\
& +\sum_{B^{\prime} \in P\left(\sigma_{j} \backslash\left(\sigma_{i} \backslash\{b\}\right)\right)}\left(l_{B^{\prime}}\left(h_{B^{\prime}}\right)-l_{B^{\prime} \cup\{b\}}\left(h_{B^{\prime} \cup\{b\}}\right)\right) s\left(B^{\prime}\right) f\left(\sigma_{j} \backslash B^{\prime}\right) \\
& +\sum_{B^{\prime} \in P\left(\sigma_{j} \backslash\left(\sigma_{i} \backslash\{b\}\right)\right)} \sum_{\Omega \in P\left(\sigma_{i} \cap \sigma_{j}\right)}\left(l_{B^{\prime} \cup \Omega}\left(h_{B^{\prime} \cup \Omega}\right)-l_{B^{\prime} \cup \Omega \cup\{b\}}\left(h_{B^{\prime} \cup \Omega \cup\{b\}}\right)\right) \\
& +\sum_{A^{\prime} \in P\left(\left(\sigma_{i} \backslash\{b\}\right) \backslash \sigma_{j}\right)}\left(l_{A^{\prime} \cup\{b\}}\left(h_{A^{\prime} \cup\{b\}}\right)-l_{A^{\prime}}\left(h_{A^{\prime}}\right)\right) s\left(B^{\prime}\right) f\left(\left(\sigma_{i} \backslash\{b\}\right) \backslash A^{\prime}\right) \\
& \sum_{A^{\prime} \in P\left(\left(\sigma_{i} \backslash\{b\}\right) \backslash \sigma_{j}\right)}\left(\sum_{\Omega \in P\left(\sigma_{i} \cap \sigma_{j}\right)}\left(l_{A^{\prime} \cup \Omega \cup\{b\}}\left(h_{A^{\prime} \cup \Omega \cup\{b\}}\right)-l_{A^{\prime} \cup \Omega}\left(h_{A^{\prime} \cup \Omega}\right)\right)\right. \\
& \left.\times s\left(A^{\prime} \cup \Omega\right) f\left(\left(\sigma_{i} \backslash\{b\}\right) \backslash\left(A^{\prime} \cup \Omega\right)\right)\right] .
\end{aligned}
$$

Let $X \subseteq E$ represent any set of SPs (even empty). Then,

- $f(X), s(X) \geq 0$ (as probabilities);

- $f\left(\sigma_{j} \backslash X\right)-f\left(\left(\sigma_{i} \backslash\{b\}\right) \backslash X\right)>0$ (follows from $\left.\left|\sigma_{i}\right|>\left|\sigma_{j}\right|+1\right)$;

- $W \geq l\left(h_{b}\right)($ since $\sigma \in N E$, by Claim $4.3(\mathrm{i}))$;

- $l_{X}\left(h_{X}\right) \geq l_{X \cup\{b\}}\left(h_{X \cup\{b\}}\right)$ (since $\left.X \subseteq X \cup\{b\}\right)$;

- from the choice of $b$, for all $e \in \sigma_{i} \backslash \sigma_{j}, l\left(h_{e}\right) \leq l\left(h_{b}\right)$

$\Rightarrow$ for all $Y \in P\left(\sigma_{i} \backslash \sigma_{j}\right)$ (and, in particular, for $Y \in P\left(\left(\sigma_{i} \backslash\{b\}\right) \backslash \sigma_{j}\right)$ ), $l_{Y \cup X \cup\{b\}}\left(h_{Y \cup X \cup\{b\}}\right)=l_{Y \cup X}\left(h_{Y \cup X}\right)$. 
Therefore, we finally get $\left[\pi_{i}(\sigma)+\pi_{j}(\sigma)\right]-\left[\pi_{i}(\hat{\sigma})+\pi_{j}(\hat{\sigma})\right] \geq 0$.

Proof of Claim 5.4: For agent $i$, the inequality holds because $\sigma$ is the Nash equilibrium strategy profile, satisfying $\pi_{i}(\sigma) \leq \pi_{i}\left(\sigma_{1}, \ldots, \hat{\sigma}_{i}, \ldots, \sigma_{n}\right)$, for all $\hat{\sigma}_{i} \in \Sigma_{i}$. For other agents, the inequality follows from the fact that the addition of SP $e$ to the agent $i$ 's strategy increases the congestion of this SP and does not change the congestion of the others. Then, the disutility of any agent using SP $e$ increases, and the disutility of any agent that does not use this $\mathrm{SP}$ is the same.

Proof of Proposition 5.5: First we prove that the combination of strategies $\sigma$ described above is a Nash equilibrium strategy profile. Let $i$ be an agent playing $\sigma_{i}=E$. We want to show that for any $\hat{\sigma}_{i} \in \Sigma_{i}, \pi_{i}(\sigma) \leq \pi_{i}\left(\sigma_{1}, \ldots, \hat{\sigma}_{i}, \ldots, \sigma_{n}\right)$.

The disutility of agent $i$ for $\sigma$ is

$$
\begin{aligned}
\pi_{i}(\sigma) & =W f(E)+\sum_{A \in P(E)} l_{A}\left(h_{A}^{\sigma}\right) s(A) f(E \backslash A) \\
& =W f^{m}+l\left(h^{* *}\right) \sum_{A \in P(E)} s(A) f(E \backslash A)=W f^{m}+l\left(h^{* *}\right)\left(1-f^{m}\right) .
\end{aligned}
$$

The disutility of agent $i$ for $\hat{\sigma}_{i}$ is

$$
\pi_{i}\left(\sigma_{1}, \ldots, \hat{\sigma}_{i}, \ldots, \sigma_{n}\right)=W f^{q}+l\left(h^{* *}\right)\left(1-f^{q}\right),
$$

where $q=\left|\hat{\sigma}_{i}\right|(0 \leq q \leq m)$.

Then, $\pi_{i}\left(\sigma_{1}, \ldots, \hat{\sigma}_{i}, \ldots, \sigma_{n}\right)-\pi_{i}(\sigma)=W f^{q}+l\left(h^{* *}\right)\left(1-f^{q}\right)-W f^{m}+l\left(h^{* *}\right)\left(1-f^{m}\right)$

$$
=\left(f^{q}-f^{m}\right)\left(W-l\left(h^{* *}\right)\right) \geq 0 .
$$

Let $j$ be an agent playing $\sigma_{j}=\varnothing$. We want to show that $\pi_{j}(\sigma) \leq \pi_{j}\left(\sigma_{1}, \ldots, \hat{\sigma}_{j}, \ldots, \sigma_{n}\right)$, for any $\hat{\sigma}_{j} \in \Sigma_{j}$.

$$
\begin{aligned}
\pi_{j}\left(\sigma_{1}, \ldots, \hat{\sigma}_{j}, \ldots, \sigma_{n}\right)-\pi_{j}(\sigma) & =W f^{q}+l\left(h^{* *}+1\right)\left(1-f^{q}\right)-W \\
& =\left(1-f^{q}\right)\left(l\left(h^{* *}+1\right)-W\right) \geq 0 .
\end{aligned}
$$

By (56) and (57), $\sigma$ is a Nash equilibrium combination of strategies. By Claims 5.3 and 5.4, this is the worst equilibrium strategy profile. 